\title{
Continuous-time Delegated Portfolio Management with Homogeneous Expectations: \\ Can an Agency Conflict Be Avoided?
}

First version: December 9, 2004

This version: December 2, 2005

\author{
Holger Kraft \\ University of Kaiserslautern, Department of Mathematics, \\ Mathematical Finance Group, 67653 Kaiserslautern, Germany, \\ email: kraft@mathematik.uni-kl.de \\ phone: +496312053876 \\ and \\ Fraunhofer ITWM, Institute for Industrial Mathematics, \\ Department of Finance, Kaiserslautern, Germany
}

\section{Ralf Korn}

University of Kaiserslautern, Department of Mathematics, Mathematical Finance Group, 67653 Kaiserslautern, Germany

and

Fraunhofer ITWM, Institute for Industrial Mathematics, Department of Finance, Kaiserslautern, Germany 


\section{Continuous-time Delegated Portfolio Management with Homogeneous Expectations: Can an Agency Conflict Be Avoided?}

ABSTRACT: In a continuous-time framework, the issue of how to delegate an investor's portfolio decision to a portfolio manager is studied. Firstly, we solve the first-best problem where the investor is able to force the manager to implement a certain strategy. For the second-best case, a specific quadratic contract is introduced resolving the agency conflict completely in the sense that the solutions to the first-best and second-best problems coincide. This contract can be implemented if the investor is able to observe the value of the growth optimal portfolio at her investment horizon. Consequently, this portfolio serves as a perfect benchmark. Instead of the quadratic contract, one can also use a contract containing a suitable exchange option. If the investment opportunity set is assumed to be constant, in equilibrium the value of the market portfolio is a sufficient statistic for the value of the growth optimal portfolio. Hence, in this situation, even a portfolio with a constant number of assets (passive index) can replace the growth optimal portfolio in the quadratic contract. Throughout the paper we assume both the investor and the manager to have homogeneous expectations about the investment opportunity set, i.e. both individuals are equally well informed about the parameters of the asset price dynamics. This, however, does not necessarily mean that investor and manager are symmetrically informed about all prices.

KeYwords: delegated portfolio decision, Merton's portfolio problem, principal-agent theory, quadratic contract, exchange option, growth optimal portfolio 


\section{Introduction}

Delegating investment decisions to portfolio managers is an important issue in practice and has attracted the interest of researchers over decades. There exist at least two different strands of literature dealing with this topic: ${ }^{1}$ The first strand considers delegated portfolio problems where the portfolio manager (he) has private information about the market or at least about some traded securities. To phrase it differently, the manager is assumed to have private information about the investor's opportunity set. For example, one can assume the manager to possess superior predictability of stock returns. Papers in this direction include Admati/Pfleiderer (1997), Bhattacharya/Pfleiderer (1985), Brennan (1993), Heinkel/Stoughton (1994), Kihlstrom (1988), Dybvig/Farnsworth/Carpenter (2001), Stoughton (1993), and Zender (1987), among others. However, people often delegate their portfolio decisions to managers without necessarily believing that these managers possess private information. In other words, investor and manager have homogeneous expectations about the investment opportunity set. The canonical example is a passive fund trying to track a given benchmark such as Dow Jones, Nasdaq 100, or S\&P 500. Papers in this direction include Ou-Yang (2003), Cadenillas et al. (2004), and Cvitanic et al. (2004). There are several reasons to delegate portfolio decisions to professionals even if no private information is present: For instance, the investor (she) may not be able to follow the market as closely as the managers. Furthermore, if the investor's wealth is not sufficiently big, she may not be able to hold a well-diversified portfolio without resorting to mutual funds. This does not mean that information asymmetries do not exist in those problems. Actually it is reasonable to assume that investor and manager are not equally wellinformed about market prices. However, the manager has no private information in the sense that he knows something which other professional market participants do not know. To characterize these two kinds of information asymmetries, the first kind is said be the case with private information and the second kind is said to be the case where the investor suffers from a lack of (in principle publicly available) information.

For several reasons, it is crucial to strictly distinguish between these settings. The case with private information corresponds to a situation where the investor buys actively managed funds, whereas the case with lack of information refers to a situation where the investor delegates her portfolio decision to the manager of a passively managed fund without expecting superior returns (compared to the corresponding benchmark). In the case with private information the investor expects the manager to beat a benchmark, whereas in the second case the investor expects the manager to track a benchmark as closely as possible. From a theoretical point of view, this may lead to completely different results about how to relate the manager's compen-

\footnotetext{
${ }^{1}$ Apart from these strands, there are also papers dealing with the effects of option-like contracts on the manager's portfolio decision. The design of the manager's contract is taken as exogenously given. See, e.g., the insightful paper by Ross (2004) and the references therein.
} 
sation to some benchmark or if this is reasonable at all. For instance, in a model with a privately informed manager, Admati/Pfleiderer (1997) show that the use of benchmarks such as stock indices may lead to inferior results. In contrast, this paper (as well as the papers of Ou-Yang (2003) and Cvitanic et al. (2004)) give strong evidence that benchmarks reduce the agency conflict if no private information exists. The conclusion of Ou-Yang (2003) that this is due to the fact that he also allows for symmetric contracts (among other reasons) may be misleading because he compares his results with the implications of models which are tailored to different settings. In our paper it is shown that a certain type of an asymmetric contract leads to the same results as in Ou-Yang (2003), but even for a broader class of utility functions. As a matter of fact, it may be misleading to conclude from a situation without private information that the same results should hold in situations with private information. We will come back to this point later on.

As mentioned above, our paper is about the case without private information and it is thus crucial how severe the investor's lack of information is assumed to be. We distinguish three cases each representing a specific level of information. These cases are presented in the order of increasing level of information (decreasing level of information asymmetry between manager and investor):

(1) Uninformed investor: In such a setting the investor is only able to observe the portfolio value at the end of the investment period. This is a somewhat unrealistic view of the investor's ability to gather information.

(2) Partially informed investor: Here the investor is able to observe the value of the portfolio as well as prices of some securities or values of benchmark portfolios at the end of the investment period. Of course, one can distinguish several subcases depending on the number of observable prices or benchmark portfolios.

(3) Fully informed investor: This investor has all information about the security prices including all possible benchmarks which can be constructed from the traded securities.

The investor can only base the contractual arrangements of the fee on those variables which she is able to observe. We emphasize that all three cases are second-best problems in the sense that the investor is not able to monitor the portfolio weights chosen by the agent (his trading record), i.e. she is not able to offer the manager a forcing contract based on the chosen portfolio weights. If this is also possible, then we are in a first-best situation where the investor can force the manager to implement a specific portfolio strategy. Table 1 summarizes the different forms of second-best problems. 


\begin{tabular}{l|l|l} 
& with Private Information & with Lack of Information \\
\hline Characterization & $\begin{array}{l}\text { Superior information of } \\
\text { the manger about the in- } \\
\text { vestor's opportunity set }\end{array}$ & $\begin{array}{l}\text { Inferior information level of } \\
\text { the investor about (publicly } \\
\text { available) security prices }\end{array}$ \\
Information level & $\begin{array}{l}\text { e.g. superior predictability } \\
\text { of returns by the manager }\end{array}$ & $\begin{array}{l}\text { 1. Uniformed investor } \\
\text { 2. Partially informed investor } \\
\text { 3. Fully informed investor }\end{array}$
\end{tabular}

Table 1: Delegated Portfolio Problems

It is now interesting to ask the following questions:

(i) Given one of the situations (1)-(3), how does the second-best optimal contract for the manager look like?

(ii) How can the investor reduce the agency conflict by gathering additional information?

(iii) Is it reasonable to include benchmarks in the compensation scheme and is there something like a perfect benchmark (at least from a theoretical point of view)?

The first question implicitly assumes that in situation (1) or (2) the investor is not able to improve her state of being less informed than the manager. Although it is important to search for second-best optimal contracts, it is also important to figure out how the investor can reduce the agency conflict gathering relevant information (i.e. information reducing the conflict). More precisely, it seems of high practical relevance to guide an investor how to distinguish between relevant and irrelevant information. Although investors may not be able (and willing) to observe all publicly available information about market prices, they may be able (and willing) to observe some of the prices if this really sets them better off. ${ }^{2}$

The contributions of this paper are manifold:

- Using a quadratic compensation scheme, we show that the agency conflict in a delegated portfolio problem with homogeneous expectations (but not necessarily without information asymmetry) can be resolved completely for all types of reasonable utility functions. To our knowledge, this specific contract design has not been applied to settings with homogeneous expectations. Providing a five line proof (see proof of Proposition 5.1, (i)), the mathematical technicalities needed to prove optimality in the second-best problem are re-

\footnotetext{
${ }^{2}$ In the classical principal-agent setting, this issue is for instance addressed by Holmstrom (1979) who assumes collecting of information to be costly. Note that in contrast to his setting gathering information about security prices or index values seems to be almost costless.
} 
duced to a minimum. ${ }^{3}$ Furthermore, we show that one can get the same results if the manager accepts a contract containing a suitable exchange option.

- By applying one of these contracts, one actually needs to consider the firstbest problem only. This problem is solved by generalizing an idea of Ou-Yang (2003) to use the martingale approach. In fact, this boils down to solve a Lagrangian leading to the Pareto optimal sharing rule which provides efficient risk-sharing between the two individuals present in the model. Due to the papers of Borch (1960, 1962), Wilson (1968), and Amershi/Stoeckenius (1983), among others, this is a well-studied issue. Contrary to the existing literature, we also allow for non-negativity constraints on the shares of wealth of the investor and the manager.

- We identify a perfect benchmark for our delegated portfolio problem, the wellknown growth optimal portfolio (GOP), which has several important features for pricing and ordinary portfolio optimization. Our paper demonstrates its importance for delegated portfolio management as well. ${ }^{4}$ We emphasize that observing the value of the growth optimal portfolio only at the end of the investment period is sufficient to resolve the agency conflict completely, i.e. continuous monitoring of this benchmark portfolio is not even necessary. The same is true if the investor can observe a sufficient statistic for the value of the growth optimal portfolio. If this single piece of information is available for the investor, depends on the investor's level of information and the dynamics of the traded assets.

- Assuming the financial market to be in equilibrium, we demonstrate for a setting with constant investment opportunity set that the market portfolio is a sufficient statistic for the growth optimal portfolio. In this case, a passive index can be used as benchmark as well.

The paper is structured as follows: In Section 2 the related literature is reviewed. Section 3 sets up the delegated portfolio problem with homogeneous expectations. The first-best solution is derived in Section 4, while the second-best problem is considered in Section 5. In Section 6, we analyze the implications for our setting if the financial market is in equilibrium. Technical details and most of the proofs are presented in the Appendix.

\footnotetext{
${ }^{3}$ We ask the reader to compare our proof with the proofs in Ou-Yang (2003), Cadenillas et al. (2004), and Cvitanic et al. (2004).

${ }^{4}$ Although Ou-Yang (2003) and Cvitanic et al. (2004) also mention that parts of their optimal contracts can be identified as benchmarks, they do not provide additional economic interpretations of their benchmarks. Ou-Yang (2003) only mentions that his benchmark is an "active index" meaning that the numbers of the included assets vary over time. At the end of Section 5 we will discuss this point in more detail. On the other hand, in Cvitanic et al. (2004) the benchmark is the solution to a system of forward-backward stochastic differential equations (of adjoint processes to the stochastic control problem) and no economic interpretation is provided.
} 


\section{$2 \quad$ Related Literature}

Delegated portfolio problems can be considered as principal-agent relationships where the agent controls the drift and volatility of the state process (wealth), simultaneously. Most of the papers on continuous-time principal-agent problems, however, deal with different situations. For example, Holmstrom/Milgrom (1987) or Schättler/Sung (1993) assume that the agent can control the drift only, whereas Sung (1995) assumes that the agent can control the drift and volatility independently. While these settings are very important for various applications in economics and finance, they do not apply to delegated portfolio problems. Since the focus of this paper is on delegating portfolio decisions with homogeneous expectations, we do not comment on this papers in detail and focus our discussion in this section on the papers by Ou-Yang (2003), Cadenillas et al. (2004), and Cvitanic et al. (2004). Ou-Yang (2003) considers a delegated portfolio problem being similar to ours. The investor delegates her portfolio choice to a manager possessing no superior information about the market. Contrary to our approach, throughout his paper the investment opportunity set is assumed to be constant and the manager is assumed to have exponential utility. Two settings are then analyzed: In a not necessarily complete market (the dimension of the Brownian motion may be greater than the number of risky assets) the problem is solved for an investor having exponential utility function and the following cost function at time $t$ :

$$
C(t, \pi, X)=\bar{c}+0.5 X^{2} \pi^{\prime} k(t) \pi+\lambda X,
$$

where $\bar{c}$ and $\lambda$ are constants, $\pi$ denotes the proportions of the assets held, $X$ denotes wealth, and $k$ is a deterministic matrix. The accumulated costs at time $T$ are then defined as $C(T)=\int_{0}^{T} C(s, \pi(s), X(s)) d s$. Furthermore, for a complete market setting, the problem is solved for an investor with power utility function and constant costs, i.e. $k \equiv 0$ and $\lambda \equiv 0$. An incentive conflict arises from the fact that the investor is not able to offer all kinds of contracts. Especially, some path-dependent contracts are excluded. We will come back to this point in Section 5 .

The papers by Cadenillas et al. (2004) and Cvitanic et al. (2004) seem to consider a rather general setting. They set up a model where the agent can either control the drift and volatility of wealth simultaneously or can control them independently. Since we concentrate on delegated portfolio management, comments made in our paper only refer to the first case. To derive their results, the authors need not specify certain types of utility functions which is similar to our paper. In Cadenillas et al. (2004) a Brownian and a more general semi-martingale framework is considered, whereas Cvitanic et al. (2004) focus on a Brownian framework only. Apart from the last part of Cvitanic et al. (2004), it is a standing assumption in both papers that the investor can contract upon all publicly available information at time $T$, i.e. she is fully informed corresponding to situation (3) of the introduction. It is thus not surprising that even in a semi-martingale setting the agency conflict can be resolved completely. For the same reason, their results cannot be considered as 
generalizations of Ou-Yang's who considers a much more restricted contract space. Again we will come back to this point latter on. Let us finally note that in the last part of Cvitanic et al. (2004) a setting is considered which is referred to as a hidden action case. The authors assume that the agent is only able to observe wealth, but the principal can only base his portfolio decision on wealth as well, i.e. the portfolio strategy would be a function of wealth only. This, however, seems to be an unrealistic assumption for (delegated) portfolio problems, but it may be justified in other applications.

\section{Delegated Portfolio Problem}

We assume that the manager (agent, he) has no superior information compared to the market and the investor (principal, she). Consequently, the investor is aware of her optimal strategy, but she is simply not able to implement this strategy by herself. Reasons may be a lack of time or the wish to diversify her portfolio. As usually, we distinguish between two cases: In the first-best case, ex-post she observes the portfolio strategy of the manager and pays him an adequate fee only if he has implemented her optimal strategy. Such a contract is called a forcing contract and provides Pareto optimal risk-sharing between principal and agent. In the second best case, she is not able to observe the strategy chosen by the manager and one of the three situations (i)-(iii) described in the introduction prevails. So in the second-best case there is a trade-off between risk-sharing and providing incentives for the manager. In general, one would expect this to lead to an inferior solution compared to the first-best case.

To set up our model, let $(\Omega, \mathcal{F}, \mathcal{P})$ be a probability space. On this space an $n$ dimensional Brownian motion $W$ is given and $\left\{\mathcal{F}_{t}\right\}_{t \geq 0}$ denotes the corresponding Brownian filtration. We assume investor and manager to maximize utility from terminal wealth at time $T$ with respect to utility functions $U_{A}$ and $U_{P}$. Note that in the fields of portfolio management and principal-agent theory there seems to exist a somewhat different philosophy about formulating the respective problems and about the properties of appropriate utility functions. In portfolio theory it is usually assumed that wealth cannot become negative, ${ }^{5}$ whereas in the theory of principalagent problems it is sometimes not ensured that the shares in outcome of principal and agent are non-negative. ${ }^{6}$ This last assumption can only be justified if there exists some wealth outside the model which allows the individuals to compensate losses. This assumption is also implicitly made in the papers by Ou-Yang (2003), Cadenillas et al. (2004), and Cvitanic et al. (2004). To distinguish the resulting delegated portfolio problems, the restricted one is said to be of type I and the unrestricted one of type II. The same expressions are used for the corresponding utility functions. Note that the main difference between both definitions results from

\footnotetext{
${ }^{5}$ See Merton, e.g., $(1969,1971)$

${ }^{6}$ See e.g. Holmstrom/Milgrom (1987).
} 
the different domains of these functions. Besides, the definitions are not exclusive. For instance, the exponential utility function can be interpreted as utility function of type I or II.

Definition 3.1 (Utility Function, Type I) A concave and continuously differentiable function $U:(0, \infty) \rightarrow \mathbb{R}$ is said to be a utility function (of type I) if

$$
U^{\prime}(0) \equiv \lim _{x \searrow 0} U^{\prime}(x)>0, \quad U^{\prime}(\infty) \equiv \lim _{x \nearrow \infty} U^{\prime}(x)=0
$$

Remarks. a) $U^{\prime}$ possesses a strictly decreasing inverse function $\left(U^{\prime}\right)^{-1}:\left[0, U^{\prime}(0)\right] \rightarrow$ $[0, \infty]$. The extension $V$ to all non-negative numbers is given by

$$
V \equiv\left\{\begin{array}{cl}
\left(U^{\prime}\right)^{-1}, & y \in\left[0, U^{\prime}(0)\right] \\
0, & y \geq U^{\prime}(0)
\end{array}\right.
$$

b) Recall that we have the following estimate for the utility function ${ }^{7}$

$$
U(V(y)) \geq U(x)+y(V(y)-x), \quad y \in[0, \infty), x \geq 0 .
$$

c) We define $U(0) \equiv \lim _{x \rightarrow 0} U(x)$ and $U(\infty) \equiv \lim _{x \rightarrow \infty} U(x)$.

Definition 3.2 (Utility Function, Type II) A concave and continuously differentiable function $\mathcal{U}:(l, \infty) \rightarrow \mathbb{R}, l \in[-\infty, 0]$, is said to be a utility function of type II if

$$
\mathcal{U}^{\prime}(l)=\lim _{x \searrow l} \mathcal{U}^{\prime}(x)=\infty, \quad \mathcal{U}^{\prime}(\infty)=\lim _{x \nearrow \infty} \mathcal{U}^{\prime}(x)=0
$$

Remarks. a) This definition ensures that $\left(\mathcal{U}^{\prime}\right)^{-1}$ always exists on $(0, \infty)$ and it is not necessary to extend this function artificially.

b) Note that $(2)$ is still valid because $\mathcal{U}$ is concave on $(l, \infty)$.

c) In general, one can assume that investor and manager have different lower bounds $l_{P}$ and $l_{A}$. For simplicity, we assume these bounds to be equal.

The investment opportunities include a money market account with the dynamics

$$
d M(t)=M(t) r(t) d t
$$

$M(0)=1$. Additionally, there exists a market for $n$ stocks where the dynamics of the $i$-th stock is governed by the stochastic differential equation (SDE)

$$
d P_{i}(t)=P_{i}(t)\left[\left(\mu_{i}(t) d t+\sigma_{i}(t)^{\prime} d W(t)\right]\right.
$$

\footnotetext{
${ }^{7}$ Korn (1997), p. 64.
} 
where $\mu_{i}(t)$ is a real-valued stochastic process and $\sigma_{i}(t)$ is an $n$-dimensional stochastic process with real-valued components. ${ }^{8}$ Given some self-financing portfolio strategy $\pi$ the wealth equation for this problem reads

$$
d X^{\pi}(t)=X^{\pi}(t)\left[\left(r(t)+(\mu(t)-r(t) \mathbf{1})^{\prime} \pi(t)\right) d t+\pi(t)^{\prime} \sigma(t) d W(t)\right]
$$

$X^{\pi}(0)=x_{0}>0$, with $\mu=\left(\mu_{1}, \ldots, \mu_{n}\right)$ and $\mathbf{1}=(1, \ldots, 1) \in \mathbb{R}^{n}$. It is assumed that all coefficients of the above SDEs are progressively measurable with respect to the Brownian filtration $\left\{\mathcal{F}_{t}\right\}_{t}$ and bounded which implies that the SDEs have unique solutions.

Given some strategy $\pi$ the manager has $\operatorname{costs} C$ at time $T$ which are assumed to equal

$$
C(T)=\bar{c}+\lambda \cdot X^{\pi}(T), \quad 0 \leq \lambda<1,
$$

where $\bar{c} \geq 0$ is a constant. Actually, this linear form of the costs is not a restrictive assumption as long as costs are hedgeable as, for instance, assumed in Cadenillas et al. (2004) and Cvitanic et al. (2004). In this case one could even assume the costs to be zero without loss of generality. However, since it is straightforward to include linear costs in our problem, we decided to do so. Besides, it allows to distinguish between fixed and variable costs.

The investor rewards the manager by a compensation scheme (syn. fee, fee structure, contract, sharing rule) $I$, where the payment is made at time $T$. With a slight abuse of notation we denote $I(T)$ by $I(X(T))$ if we wish to emphasize the dependency of $I$ on $X$. Besides, we suppress the dependency on $T$ whenever convenient, i.e. we then write $X^{*}$ instead of $X^{*}(T)$. In the first-best case the investor can choose both the compensation scheme and the portfolio strategy which the manager has to implement. Hence, her optimization problem reads as follows:

$$
\max _{\pi, I} \mathrm{E}\left[U_{P}\left(X^{\pi}(T)-I(T)\right)\right]
$$

subject to the constraints

$$
\begin{aligned}
\mathrm{E}\left[U_{A}(I(T)-C(T))\right] & \geq U_{A}\left(\varepsilon_{0}\right), \\
\mathrm{E}\left[H(T) X^{\pi}(T)\right] & \leq x_{0} .
\end{aligned}
$$

Here $\varepsilon_{0}$ is some positive constant and $U_{A}\left(\varepsilon_{0}\right)$ denotes the reservation utility of the manager. The process

$$
H(t)=\exp \left(-\int_{0}^{t} r(s) d s-0.5 \int_{0}^{t}\|\theta(s)\|^{2} d s-\int_{0}^{t} \theta(s)^{\prime} d W(s)\right)
$$

equals the deflator of the economy, where $\theta \equiv\left(\sigma^{-1}\right)^{\prime}(\mu-r \mathbf{1})$ stands for the market price of risk. The constraint (8) is said to be the participation constraint (PC). Requirement (9) is the budget constraint (BC) stating that the present value of

\footnotetext{
${ }^{8}$ The assumption of a complete market is made to simplify the derivations. However, since techniques to generalize the martingale approach to incomplete markets are well-established in literature, one could also consider these cases. See Section 7 for details.
} 
terminal wealth cannot exceed the initial wealth $x_{0}$. If we consider the more realistic problem with non-negativity constraints (type I), we need to add the requirements

$$
X^{\pi}(T)-I(T) \geq 0, \quad I(T)-C(T) \geq 0,
$$

which also ensure the wealth $X^{\pi}$ to be non-negative because

$$
X^{\pi}(T) \geq I(T) \geq C(T)=\bar{c}+\lambda X^{\pi}(T) \quad \Longrightarrow \quad X^{\pi}(T) \geq \frac{\bar{c}}{1-\lambda} \geq 0 .
$$

We emphasize that these conditions are redundant if $U_{P}^{\prime}(0)=U_{A}^{\prime}(0)=\infty$ as in the case of power utility functions.

In the second-best case the investor can only choose the compensation scheme $I$ and the manager implements some portfolio strategy $\pi$ which optimizes his wealth. As pointed out in the introduction, the set of admissible compensation schemes depends on the ability of the investor to gather information. We will address this point in Section 5 when we solve the second-best problem. The optimization problem of the investor is given by

$$
\max _{I} \mathrm{E}\left[U_{P}\left(X^{\pi}(T)-I\left(X^{\pi}(T)\right)\right)\right]
$$

subject to the constraints

$$
\begin{aligned}
\mathrm{E}\left[U_{A}\left(I\left(X^{\pi}(T)\right)-C(T)\right)\right] & \geq U_{A}\left(\varepsilon_{0}\right), \\
\pi & \in \arg \max _{\pi^{\prime}} \mathrm{E}\left[U_{A}\left(I\left(X^{\pi^{\prime}}(T)\right)-C(T)\right)\right], \\
\mathrm{E}\left[H(T) X^{\pi}(T)\right] & \leq x_{0} .
\end{aligned}
$$

Additionally, the non-negativity constraints (10) may be required as well. The constraint (13) is said to be the incentive constraint and its presence distinguishes the second-best from the first-best problem. Since the investor cannot monitor the portfolio strategy chosen by the manager, the manager picks out a strategy which optimizes his expected utility. Therefore, constraint (13) needs to be added to the delegated portfolio problem. Since choosing a strategy leads to specific terminal wealth, one can alternatively formulate the constraint as follows:

$$
X(T) \in \arg \max _{X^{\prime}(T)} \mathrm{E}\left[U_{A}\left(I\left(X^{\prime}(T)\right)-C(T)\right)\right] .
$$

Since the compensation scheme $I$ needs to perform two distinct tasks, we are facing a trade-off: Firstly, the compensation scheme should ensure efficient risk-sharing and, secondly, it shall give the manager the incentive to take the investor's preferences into account. Due to this trade-off the second-best optimal utility of the investor in general decreases compared to the first-best case.

\section{First-best Problem}

In this section the first-best problem (7)-(9) is solved. We start by deriving the candidates for the optimal terminal wealth and the optimal fee using the usual pointwise optimization approach introduced by Cox/Huang $(1989,1991)$ and Karatzas et 
al. (1987). For specific utility functions this approach was also applied by Ou-Yang (2003). The proof that these candidates are indeed the solutions to the problem is provided in the Appendix. Since choosing a strategy $\pi$ can be interpreted as choosing the corresponding terminal wealth $X^{\pi}(T)$, the Langrangian of the problem reads as follows:

$L\left(X, I, y_{1}, y_{2}\right)=U_{P}(X-I)+y_{1}\left\{U_{A}(I-\lambda X-\bar{c})-U_{A}\left(\varepsilon_{0}\right)\right\}+y_{2}\left\{x_{0}-H(T) X\right\}$.

This leads to the following first-order conditions:

$$
\begin{aligned}
U_{P}^{\prime}(X-I)-y_{1} \lambda U_{A}^{\prime}(I-\lambda X-\bar{c})-y_{2} H(T) & =0 \\
U_{P}^{\prime}(X-I)-y_{1} U_{A}^{\prime}(I-\lambda X-\bar{c}) & =0
\end{aligned}
$$

where the second condition ensures Pareto optimal risk-sharing between manager and investor. ${ }^{9}$ Hence, we get

$$
U_{A}^{\prime}(I-\lambda X-\bar{c})=\frac{y_{2}}{y_{1}} \frac{1}{1-\lambda} H(T)
$$

and thus the candidate for the optimal contract is given by

$$
I^{*}(T)=V_{A}\left(\frac{y_{2}}{y_{1}} \frac{1}{1-\lambda} H(T)\right)+\lambda X(T)+\bar{c} .
$$

Substituting (16) into the second first-order condition, the candidate for the principal's optimal share of wealth reads as

$$
X^{*}(T)-I^{*}(T)=V_{P}\left(y_{2} \frac{1}{1-\lambda} H(T)\right) .
$$

Solving (17) and (18) for $X^{*}$ and $I^{*}$ we obtain the optimal wealth and optimal fee. The candidate for the optimal fee is given by

$$
I^{*}(T)=\frac{1}{1-\lambda} V_{A}\left(\frac{y_{2}}{y_{1}} \frac{1}{1-\lambda} H(T)\right)+\frac{\lambda}{1-\lambda} V_{P}\left(y_{2} \frac{1}{1-\lambda} H(T)\right)+\frac{1}{1-\lambda} \bar{c} .
$$

The candidate for the optimal terminal wealth reads

$$
X^{*}(T)=\frac{1}{1-\lambda}\left\{V_{A}\left(\frac{y_{2}}{y_{1}} \frac{1}{1-\lambda} H(T)\right)+V_{P}\left(y_{2} \frac{1}{1-\lambda} H(T)\right)+\bar{c}\right\} .
$$

The ratio $y_{1} / y_{2}$ has to be chosen such that the participation constraint (8) is satisfied as equality. Substituting $I^{*}$ into (8) leads to

$$
\chi_{A}\left(\frac{y_{2}}{y_{1}}\right) \equiv \mathrm{E}\left[U_{A}\left(V_{A}\left(\frac{y_{2}}{y_{1}} \frac{1}{1-\lambda} H(T)\right)\right)\right]=U_{A}\left(\varepsilon_{0}\right),
$$

so that we can deduce the value of $y_{1} / y_{2}$. The Lagrangian multiplier $y_{2}$ has to be chosen such that the budget constraint is satisfied as equality as well:

$$
\mathrm{E}\left[H(T) X^{*}(T)\right]=x_{0}
$$

which is equivalent to

$$
\chi_{P}\left(y_{2}\right) \equiv \mathrm{E}\left[H(T) V_{P}\left(y_{2} \frac{1}{1-\lambda} H(T)\right)\right]=\underbrace{x_{0}-\mathrm{E}\left[H(T) I^{*}(T)\right]}_{\equiv \tilde{x}_{0}} .
$$

\footnotetext{
${ }^{9}$ See Borch $(1960,1962)$ and Wilson (1968).
} 
In order to obtain a reasonable setting, we assume the investor's initial wealth to be bigger than the net present value of the manager's compensation scheme implying $\tilde{x}_{0}>0$. From $y_{1} / y_{2}$ and $y_{2}$ we can finally calculate the value of $y_{1}$. Let $\mathcal{Y}_{P} \equiv \chi_{P}^{-1}$ and $\mathcal{Y}_{A} \equiv \chi_{A}^{-1}$. Imposing the usual technical assumptions one can prove the optimality of the candidates for fee and wealth:

Proposition 4.1 (Optimality) Assume assumptions (A1) through (A3) presented in the Appendix to hold. Then the optimal net payment to the agent reads as

$$
Z_{A}^{*}(T) \equiv I^{*}(T)-C(T)=V_{A}\left(y_{A}^{*} \frac{1}{1-\lambda} H(T)\right)
$$

with $y_{A}^{*}=\mathcal{Y}_{A}\left(U_{A}\left(\varepsilon_{0}\right)\right)>0$. The principal's optimal share of wealth is given by

$$
Z_{P}^{*}(T) \equiv X^{*}(T)-I^{*}(T)=V_{P}\left(y_{P}^{*} \frac{1}{1-\lambda} H(T)\right)
$$

with $y_{P}^{*}=\mathcal{Y}_{P}\left(x_{0}-\mathrm{E}\left[H(T) I^{*}(T)\right]\right)$, where the present value of the optimal fee equals

$$
\mathrm{E}\left[H(T) I^{*}(T)\right]=\lambda x_{0}+\mathrm{E}\left[H(T) Z_{A}^{*}(T)\right]+\bar{c} \mathrm{E}[H(T)] .
$$

Due to the completeness of the market there exists a unique strategy $\pi^{*}$ which replicates $X^{*}(T)$.

Proof. See Appendix.

From the above representations of the optimal fee, it is not obvious in which way this fee is related to wealth. Disregarding costs, sharing rules are linear if

$$
-\frac{U_{i}^{\prime}(z)}{U_{i}^{\prime \prime}(z)}=\rho_{i}+\kappa z
$$

where $\kappa$ and $\rho_{i}, i \in\{P, A\}$, are constants. ${ }^{10}$ This condition is satisfied for power utility functions with the same relative risk aversion parameters and exponential utility functions. However, power utility functions with different relative risk aversion parameters do not meet this condition and thus the first-best sharing rule $I^{*}$ is not affine linear in wealth. The following examples are intended to highlight the results in the first-best case. For the sake of simplicity, we consider a delegated portfolio problem where the investor and the manager both possess power utility functions. As mentioned above, the non-negative constraints are not binding in this case. Furthermore, we shortly analyze the problem if both investor and manager possess exponential utility functions and these constraints are not imposed.

Example "Power Utility". For simplicity, we assume a constant investment opportunity set implying that the coefficients of the deflator $H$ are constants. Furthermore, the utility functions of the principal and the agent are given by $U_{P}(x)=\frac{1}{\delta} x^{\delta}$ and $U_{A}(x)=\frac{1}{\gamma} x^{\gamma}$ with $\delta, \gamma \in(-\infty, 0) \cup(0,1)$. Therefore, we have $V_{A}(y)=y^{\frac{1}{\gamma-1}}$. This gives $\chi_{A}(y)=\frac{1}{\gamma}\left(\frac{1}{1-\lambda} y\right)^{\frac{\gamma}{\gamma-1}} m_{\gamma}$, where $m_{\gamma}=\mathrm{E}\left[H(T)^{\frac{\gamma}{\gamma-1}}\right]$. Hence, we obtain

$$
\mathcal{Y}_{A}(z)=(1-\lambda) \cdot\left(\frac{\gamma \cdot z}{m_{\gamma}}\right)^{\frac{\gamma-1}{\gamma}} \quad \text { and } \quad y_{A}^{*}=(1-\lambda) \cdot\left(\frac{\varepsilon_{0}}{\left(m_{\gamma}\right)^{1 / \gamma}}\right)^{\gamma-1}
$$

\footnotetext{
${ }^{10}$ See, e.g., Mossin (1973), p. $113 f f$.
} 
This leads to the following net payment to the agent

$$
Z_{A}^{*}(T)=\frac{\varepsilon_{0}}{\left(m_{\gamma}\right)^{1 / \gamma}} H(T)^{\frac{1}{\gamma-1}}
$$

The corresponding results for the principal are given by

$$
\mathcal{Y}_{P}(z)=(1-\lambda) \cdot\left(\frac{z}{m_{\delta}}\right)^{\delta-1} \quad \text { and } \quad y_{P}^{*}=(1-\lambda) \cdot\left(\frac{\tilde{x}_{0}}{m_{\delta}}\right)^{\delta-1}
$$

with $m_{\delta}=\mathrm{E}\left[H(T)^{\frac{\delta}{\delta-1}}\right]$ and $\tilde{x}_{0}=(1-\lambda) x_{0}-\bar{c} e^{-r T}-\varepsilon_{0}\left(m_{\gamma}\right)^{\frac{\gamma-1}{\gamma}}$. Note that $\tilde{x}_{0}$ can be interpreted as investor's net wealth after subtracting the present value of variable and fixed costs, $\lambda x_{0}+\bar{c} e^{-r T}$, and the present value of the agent's net share after costs, $\varepsilon_{0}\left(m_{\gamma}\right)^{\frac{\gamma-1}{\gamma}}$.

Thus the net payment to the principal equals

$$
Z_{P}^{*}(T)=\frac{\tilde{x}_{0}}{m_{\delta}} H(T)^{\frac{1}{\delta-1}}
$$

which leads to the following optimal wealth

$$
X^{*}(T)=\underbrace{\frac{1}{1-\lambda} \frac{\varepsilon_{0}}{\left(m_{\gamma}\right)^{1 / \gamma}} H(T)^{\frac{1}{\gamma-1}}}_{=: X^{(1)}(T)}+\underbrace{\frac{1}{1-\lambda} \frac{\tilde{x}_{0}}{m_{\delta}} H(T)^{\frac{1}{\delta-1}}}_{=: X^{(2)}(T)}+\underbrace{\frac{1}{1-\lambda} \bar{c}}_{=: X^{(3)}(T)} .
$$

Hence, the optimal wealth consists of two random parts (one for the agent and one for the principal) and a constant part which can be attained by the following replication strategy:

- Replicate $H(T)^{\frac{1}{\gamma-1}}$ with an initial capital of $\frac{1}{1-\lambda} \varepsilon_{0}\left(m_{\gamma}\right)^{\frac{\gamma-1}{\gamma}}$.

- Replicate $H(T)^{\frac{1}{\delta-1}}$ with an initial capital of $\frac{1}{1-\lambda} \tilde{x}_{0}$.

- Buy a zero-coupon bond with notional $\frac{1}{1-\lambda} \bar{c}$ maturing at $T$.

This leads to the following optimal portfolio strategy which the agent has to implement: ${ }^{11}$

$$
\pi^{*}(t)=\left\{\frac{1}{1-\gamma} \frac{X^{(1)}(t)}{X^{*}(t)}+\frac{1}{1-\delta} \frac{X^{(2)}(t)}{X^{*}(t)}\right\} \sigma^{-1} \theta .
$$

Hence, we obtain a value-weighted combination of Merton strategies. Note that $X^{(1)}(t)$ and $X^{(2)}(t)$ are given by the present values

$$
\begin{aligned}
X^{(1)}(t) & =\frac{1}{1-\lambda} \frac{\varepsilon_{0}}{\left(m_{\gamma}\right)^{1 / \gamma}} H(t)^{-1} \mathrm{E}\left[H(T)^{\frac{\gamma}{\gamma-1}} \mid \mathcal{F}_{t}\right] \\
X^{(2)}(t) & =\frac{1}{1-\lambda} \frac{\tilde{x}_{0}}{m_{\delta}} H(t)^{-1} \mathrm{E}\left[H(T)^{\frac{\delta}{\delta-1}} \mid \mathcal{F}_{t}\right] .
\end{aligned}
$$

Here, $X^{(1)}$ can be interpreted as the present value of the investor's net share after costs and $X^{(2)}$ as the present vlaue of the manager's net share after fixed costs, but before variable costs. Due to the fixed costs, $X^{(1)}(t)$ and $X^{(2)}(t)$ do not add up to the optimal wealth $X^{*}(t)$. Of course, we can also assume fixed costs to be

\footnotetext{
${ }^{11}$ See, e.g., Korn (1997), pp. 75ff, for a suitable representation result.
} 
paid upfront without affecting the general results. Then the manager allocates net wealth after fixed costs being defined by $\hat{X}(0)=X(0)-\bar{c} e^{-r T}$ and the optimal strategy reads

$$
\pi^{*}(t)=\left\{\frac{1}{1-\gamma} \frac{X^{(1)}(t)}{\hat{X}^{*}(t)}+\frac{1}{1-\delta} \frac{X^{(2)}(t)}{\hat{X}^{*}(t)}\right\} \sigma^{-1} \theta,
$$

where $\hat{X}^{*}(t)=X^{(1)}(t)+X^{(2)}(t)$ is the optimal net wealth after fixed costs.

Example "Exponential Utility". Assume manager and investor to have exponential utility functions of the form $U_{A}(x)=-\frac{1}{\gamma} e^{-\gamma x}$ and $U_{A}(x)=-\frac{1}{\delta} e^{-\delta x}$ with $\gamma, \delta>0$. This implies $V_{A}(y)=-\frac{1}{\gamma} \ln (y)$ and $V_{P}(y)=-\frac{1}{\delta} \ln (y)$. Solving (20) for $\ln (H(T))$ and substituting into (19) leads to

$$
I^{*}(T)=\frac{\delta+\lambda \gamma}{\delta+\gamma} X^{*}(T)+\frac{\ln \left(y_{1}\right)}{\delta+\gamma},
$$

where the Lagrangian multiplier $y_{1}$ needs to be determined such that the participation constraint is satisfied. As indicated above, the Pareto optimal sharing rule is affine linear in wealth. We now take a different perspective and consider what will happen to the utility functionals of manager and investor if the investor chooses a contract of the form

$$
I(T)=\frac{\delta+\lambda \gamma}{\delta+\gamma} X(T)+\text { const }
$$

Then we obtain

$$
\mathrm{E}\left[U_{i}(X(T)-I(T))\right]=-k_{i} \mathrm{E}\left[\exp \left(-\frac{\delta \gamma(1-\lambda)}{\delta+\gamma} X(T)\right)\right]
$$

with $i \in\{A, P\}$ and $k_{i}>0$, i.e. except for positive scalars being irrelevant for decision making the utility functionals are identical. Therefore, the linear contract (24) aligns the manager's preferences with the investor's and the manager acts as if both manager and investor would be the same individual. It is thus obvious that even in the second-best case no incentive conflict will arise if the manager accepts this contract.

\section{Second-best Problem}

In the second-best case, the investor is not able to force the manager to implement a particular investment strategy. She can control the manager only indirectly via a clever choice of the compensation scheme. Once manager and investor have agreed upon this scheme, the manager cannot be controlled and takes the investment decision which optimizes his portfolio problem without taking the investor's preferences into account. This is reflected in the incentive constraints (13) or (15), respectively, which usually makes solving the second-best problem far more complicated than solving the first-best problem. In the introduction it was pointed out that even in the second-best situation there are at least three different forms of informed investors possible. If the investor is uninformed, she can only contract upon wealth. 
On the other hand, if she is fully informed, the fee can depend on all observable asset prices at time $T$, but also on contingent claims whose payoffs at time $T$ can be attained by dynamic (possible path-dependent) replication strategies. For this reason, it is a crucial point between how many different events (states of the world) the investor is able to distinguish at time $T$. The uninformed investor is only able to distinguish between events depending on final wealth. Especially, the investor cannot base the agent's fee on reasonable benchmarks because final wealth cannot serve as its own benchmark. The fully informed investor, however, can distinguish between all possible events and can thus offer sophisticated contracts. The partially informed investor lies somewhere in between both extreme cases. From a formal point of view, one can characterize the different situations via assumptions on the measurability of the contracts which the principal is allowed to offer and/or the functional form of the contracts. The set of all admissible contracts is said to be the contract space. For instance, the uninformed investor can only offer contracts being measurable w.r.t. the sigma-algebra generated by $X(T)$, whereas the informed investor is able to offer contracts being measurable w.r.t. to the sigma-algebra $\mathcal{F}_{T}$ generated by the Brownian motion driving the price processes. The papers of Cadenillas et al. (2004) and Cvitanic et al. (2004) assume that contracts are $\mathcal{F}_{T}$-measurable, i.e. they do not restrict the contract space. The paper by Ou-Yang (2003), however, allows for all kinds of contracts depending on final wealth as well as the asset prices at time $T$, but it does not allow for all kinds of path-dependent contracts. More precisely, contracts of the following form are considered:

$$
\begin{aligned}
\tilde{I}(T)= & \mathcal{E}_{T}+\int_{0}^{T} \delta_{0}(t, X(t), P(t)) d t \\
& +\int_{0}^{T} \delta_{1}(t, X(t), P(t)) d X(t)+\int_{0}^{T} \delta_{2}(t, X(t), P(t)) d P(t),
\end{aligned}
$$

where $\mathcal{E}_{T}$ is a function depending on $T, P(T)$, and $X(T)$ and $\delta_{i}$ are functions as well. ${ }^{12}$ Although otherwise stated in the papers by Cadenillas et al. (2004) and Cvitanic et al. (2004), it is therefore questionable if these two papers can be considered as generalizations of the work by Ou-Yang (2003) because their state spaces are assumed to be unrestricted.

In this paper, we take a different approach as taken in the above-mentioned papers. Instead of solving the second-best problem for the optimal contract by applying stochastic control theory or convex dual methods, we define two types of suitable contracts and show under which conditions these are the optimal contracts for our problem. We emphasize that these conditions are the same for both contracts and that they are satisfied in the above-mentioned three papers. The first one is a

\footnotetext{
${ }^{12}$ See equation (2) in his paper. Ou-Yang (2003) does not impose additional restrictions on these functions. He only requires $\mathcal{E}_{T}$ to be a "general function" of $T$, wealth at time $T$, and the asset prices at time $T$.
} 
quadratic contract and reads as follows: ${ }^{13}$

$$
I(T)=I^{F B}(T)-\left(X(T)-X^{F B}(T)\right)^{2},
$$

$I^{F B}(T)$ denotes the first-best optimal fee and $X^{F B}$ denotes the optimal wealth in the first-best problem which can be attained by the strategy $\pi^{F B}$. Hence, the first part, $I^{F B}(T)$, provides efficient risk-sharing, whereas the second part, $(X(T)-$ $\left.X^{F B}(T)\right)^{2}$, is a penalty term which punishes the agent if final wealth is different from $X^{F B}$. Note that the manager cannot control the first part. Although optimal from a theoretical point of view, it may be considered as an undesirable feature of the quadratic contract that the manager is penalized even if he outperforms the first-best solution. For this reason, we also consider a contract where the penalty part consists of an exchange option (exchange option contract):

$$
I(T)=I^{F B}(T)-\max \left\{X^{F B}(T)-X^{\pi}(T), 0\right\} .
$$

The following proposition shows the optimality of both contracts:

Proposition 5.1 (Optimal Fees) Assume that the investor is able to gather all relevant information necessary to offer the above contracts. Then the following is valid:

(i) The quadratic sharing rule (26) is optimal in the second-best problem (11). The second-best solution is then equal to the first-best solution of the problem and thus risk-sharing is Pareto optimal.

(ii) The same results are valid if the contract (27) is applied.

Proof. (i) The proof is straightforward. Assume that the manager implements a portfolio strategy $\pi$ leading to a final wealth with $P\left(X^{\pi}(T) \neq X^{F B}(T)\right)>0$, then we obtain:

$$
\begin{aligned}
\mathrm{E}\left[U_{A}(I(T)-C(T))\right] & =\mathrm{E}\left[U_{A}\left(I^{F B}(T)-C(T)-\left(X^{\pi}(T)-X^{F B}(T)\right)^{2}\right)\right] \\
& <\mathrm{E}\left[U_{A}\left(I^{F B}(T)-C(T)\right)\right]=U_{A}\left(\varepsilon_{0}\right)
\end{aligned}
$$

Hence, this constraint would be violated and thus the agent chooses $\pi=\pi^{F B}$.

(ii) Again assume that the manager implements a portfolio strategy $\pi$ leading to a final wealth with $P\left(X^{\pi}(T) \neq X^{F B}(T)\right)>0$. Firstly, note that a strategy with $X^{\pi}(T) \geq X^{F B}(T)$ and $P\left(X^{\pi}(T)>X^{F B}(T)\right)>0$ would contradict the first-best optimality of $X^{F B}(T)$ and, therefore, cannot exist. Hence, any strategy with $P\left(X^{\pi}(T) \neq X^{F B}(T)\right)>0$ implies $P\left(X^{\pi}(T)<X^{F B}(T)\right)>0$ and thus $P\left(\max \left\{X^{F B}(T)-X^{\pi}(T), 0\right\}>0\right)>0$. Replacing $\left(X^{\pi}(T)-X^{F B}(T)\right)^{2}$ by $\max \left\{X^{F B}(T)-X^{\pi}(T), 0\right\}$, the inequality (28) still holds.

Some points need to be addressed now: Firstly, both contracts are not forcing contracts in the classical sense because no information is needed about the strategy

\footnotetext{
${ }^{13}$ Note that in delegated portfolio problems with private information quadratic contracts are also used to give the manager the incentive to reveal his private information truly. See e.g. Bhattacharya/Pfleiderer (1985) and Stoughton (1993).
} 
implemented by the manager. An example for a forcing contract would be the following sharing rule:

$$
I(T)=I^{F B}(T)-\int_{0}^{T}\left(\pi(s)-\pi^{F B}(s)\right)^{2} d s .
$$

The crucial point is that every attainable terminal wealth $X(T)$ is uniquely connected with a trading strategy $\pi$. Therefore, forcing the manager to implement a specific trading strategy is equivalent to forcing the manager to attain a specific final wealth. Note that this remains valid in incomplete market because attainable claims are still uniquely connected with a certain trading strategy. Even if there are several trading strategies leading to the same final wealth, the investor only cares about wealth and actually does not care about the implemented trading strategy. ${ }^{14}$ This property shows the fundamental difference between delegated portfolio problems without private information and classical principal agent problems such as analyzed by Ross (1973), Mirlees (1974, 1976), Holmstrom (1979), Shavell (1979), and Holmstrom/Milgrom (1987), to mention only a few papers. In these classical papers, the agent chooses some action (effort) $a$ influencing the output $Y$ of a firm, say. However, there is no one-to-one correspondence between $a$ and $Y$, but it is assumed that, for instance, these variables are related via $Y=a+\varepsilon$ with $\varepsilon$ being noise. Since $\varepsilon$ is not observable, in the second-best case the principal is not able to deduce the effort $a$ chosen by the agent from the realized output $Y(a)$. For delegated portfolio problems it is a different story: Although final wealth is stochastic in delegated portfolio problems, the observed final wealth together with a suitable benchmark portfolio is a sufficient statistic for the portfolio strategy. Whereas in the classical setting such benchmarks are usually not available, in delegated portfolio management it is not unrealistic to assume that information about certain benchmark portfolios can be collected.

Secondly, the above contracts are not the only contracts reducing agency costs to zero. For example, every contract of the form

$$
I(T)=I^{F B}(T)-\beta\left(X(T)-X^{F B}(T)\right)^{k},
$$

where $\beta>0$ and $k=2 n$ with $n \in \mathbb{I N}$ can be applied to prove Proposition 5.1. On the other hand, one can also use a digital exchange option leading to some constant penalty $k>0$ if the manager underperforms the first-best solution: ${ }^{15}$

$$
I(T)=I^{F B}(T)-k 1_{\left\{X^{F B}(T)-X^{\pi}(T)>0\right\}} .
$$

We emphasize that our idea works for every compensation scheme $I$ with $I(T)=$ $I^{F B}(T)$ on the set $\left\{X^{F B}(T) \leq X^{\pi}(T)\right\}$ and $I(T)<I^{F B}(T)$ on the set $\left\{X^{F B}(T)>\right.$ $\left.X^{\pi}(T)\right\}$. This lack of uniqueness is, however, only valid from the ex-ante point of view. Ex-post, the actual payment is only based on the first-best sharing rule

\footnotetext{
${ }^{14}$ If costs depend on the chosen trading strategy, then obviously the manager would choose the trading strategy leading to minimal costs.

${ }^{15} 1_{A}$ denotes an indicator function being one if the event $A$ occurs and 0 else.
} 
$I^{F B}(T)$ because the manager chooses the strategy such that the penalty term is zero. For this reason, at least the ex-post sharing rule is uniquely determined.

Thirdly, it is important to realize that given the manager accepts one of the above contracts he is indifferent between maximizing his utility and minimizing the variance of the tracking error:

$$
\min _{X(T)} \operatorname{Var}\left[X(T)-X^{F B}(T)\right] .
$$

In contrast to Roll (1992), however, no restrictions on the expected tracking error are imposed implying the minimum to be always zero.

A crucial question remains to be addressed: Which information does the investor actually needs to implement the contracts? Stated differently we wish to answer the question what the term "relevant" in the assumption of Proposition 5.1 means. To provide an answer, we substitute the first-best sharing rule and the optimal wealth of the first-best problem into our quadratic contract:

$$
\begin{aligned}
I(T)= & I^{F B}(T)-\left(X(T)-X^{F B}(T)\right)^{2} \\
= & \frac{1}{1-\lambda} V_{A}\left(\frac{y_{2}}{y_{1}} \frac{1}{1-\lambda} H(T)\right)+\frac{\lambda}{1-\lambda} V_{P}\left(y_{2} \frac{1}{1-\lambda} H(T)\right)+\frac{1}{1-\lambda} \bar{c} \\
& -\left(X(T)-\frac{1}{1-\lambda}\left\{V_{A}\left(\frac{y_{2}}{y_{1}} \frac{1}{1-\lambda} H(T)\right)+V_{P}\left(y_{2} \frac{1}{1-\lambda} H(T)\right)+\bar{c}\right\}\right)^{2}
\end{aligned}
$$

Apart from final wealth $X(T)$, the only random variable being involved is obviously $H(T)$ or alternatively stated $1 / H(T)$. Hence, the investor is able to offer the quadratic contract if she can observe the value of the growth optimal portfolio at the final time $T$ which in turn can be considered as a perfect benchmark. This result which holds for the contract (27) as well is summarized in the following proposition:

Proposition 5.2 (Growth Optimal Portfolio) If the value of the growth optimal portfolio (or some sufficient statistic for the GOP) can be observed at time T, then the agency conflict is solved in the sense that the solutions to the first-best and second-best problems coincide.

As a consequence from Proposition 5.2, the sharing rule in the first-best case and the ex-post sharing rule in the second-best case coincide as well. This means especially that we will have affine linear ex-post sharing rules given condition (23) is satisfied.

Whether the value of the growth optimal portfolio can be observed depends on the assumptions about the investor's ability to gather information as well as the choice of the model. Clearly, if one assumes (as in Cadenillas et al. (2004) and Cvitanic et al. (2004)) the investor to be "informed about everything", i.e. the investor has all information contained in $\mathcal{F}_{T}$, then $H(T)$ is observable and the investor can offer the quadratic contract or the exchange option contract. This is the reason why the agency conflict disappears in the papers by Cadenillas et al. (2004) and Cvitanic et al. (2004). On the other hand, Ou-Yang (2003) assumes the investor's opportunity set to be constant. Therefore, the asset prices at final time $T$ are a sufficient statistic for the value of the GOP. Since his admissible fees (25) contain 
functions of the type $\mathcal{E}_{T}$, this assumption also ensures that our above contracts are admissible in his model and the agency conflict is solved. Note that, in the light of the Amendment to the Investment Advisors Act of 1940, Ou-Yang wishes to exclude asymmetric contracts from his contract space. However, since the function $\mathcal{E}_{T}$ is present in the representation of an admissible fee, he does not exclude asymmetric contracts depending only on the final value of some benchmark portfolio whose value can be recovered from the asset prices at time $T$ and final wealth. Furthermore, taking a closer look at the optimal contract (29) and the contract (25) the following result is obvious:

Corollary 5.1 (Shrinking the Contract Space (25)) Assume the investment opportunity set to be constant. If costs are given by (6), then without loss of generality the functions $\delta_{i}, i=0,1,2$, of the contract (25) can be chosen to be zero.

Consequently, the functions $\delta_{i}$ are only meaningful if the cost structure is more involved. In such a case, these functions can be used to take such a cost structure into account. This in turn implies that interpreting any of the three last terms in (25) as benchmarks may be misleading. Confusion about the interpretation of these three terms may stem from the fact that the decomposition of an admissible contract (25) into its four building blocks is not unique. For example, from Ito's lemma we obtain

$$
P(T) T=\int_{0}^{T} P(t) d t+\int_{0}^{T} t d P(t)
$$

where, for simplicity, we have assumed $P$ to be one-dimensional. Hence, it is equivalent to include $P(T) T$ in $\mathcal{E}_{T}$ or to include $P(t)$ in $\delta_{0}$ and $t$ in $\delta_{2}$.

\section{Equilibrium and Benchmark}

It is market practice to use benchmark portfolios as yardsticks for portfolio management. For example, an active management fund investing in US blue chips could be evaluated by comparing its performance with the performance of the Dow Jones or the S\&P 500 index. The manager of an actively managed fund will then try to outperform the relevant benchmark by forming a portfolio deliberately deviating from its benchmark portfolio. In single period models such as EV-portfolio models one cannot take into consideration that managers of actively managed funds can continuously rebalance their portfolios. In multiperiod models, however, it becomes clear that indices are passive in the sense that the numbers of their assets are held constant over time (accept for institutional issues such as dividends or changes in the indices). From this point of view, a benchmark strategy implying frequent rebalancing is an active strategy sometimes referred to as "active index". From a theoretical point of view, one could clearly argue that using a ex-ante EV-efficient strategy as benchmark is also in some sense a passive strategy, but we do not want to go into details here. In this section, we will show that the growth optimal portfolio can be replaced by a passive index (with constant numbers of assets) given our 
financial market is assumed to be in equilibrium and the investor's opportunity set is assumed to be constant. ${ }^{16}$

Since our line of arguments is adapted from Merton (1973), we keep the derivation short and refer the reader to this paper for further details. We assume that apart from our investor and manager there may be other individuals holding shares of assets, but for our further considerations we need not to specify their utility functions. Without loss of generality, one can assume that these individuals do not delegate their portfolio decisions. As in the previous sections, there are $n$ traded assets and $N_{i}$ denotes the total net supply of asset $i, i=1, \ldots, n$. Since the investor's opportunity set is assumed to be constant, each individual is indifferent between choosing portfolios from among the $n$ assets and the money market account or from two funds, where the first fund invests in the money market account and the second invests in the tangency portfolio

$$
\pi^{T}=\frac{\left(\sigma \sigma^{\prime}\right)^{-1}(\mu-r \mathbf{1})}{\aleph},
$$

$\aleph \equiv \mathbf{1}^{\prime}\left(\sigma \sigma^{\prime}\right)^{-1}(\mu-r \mathbf{1})$, consisting of risky assets only, i.e. $\mathbf{1}^{\prime} \pi^{T}=1$. Assume now the market to be in equilibrium and the money market account to be in zero net supply. Then the tangency portfolio is identical to the market portfolio consisting of all risky assets. The total market value of all risky assets equals $X^{M}=\sum_{i=1}^{n} N_{i} P_{i}$. By (30), the dynamics of the market portfolio are given by

$$
d X^{M}(t)=X^{M}(t)\left[\left(r+\left(\pi^{T}\right)^{\prime}(\mu-r \mathbf{1})\right) d t+\left(\pi^{T}\right)^{\prime} \sigma d W(t)\right]
$$

leading to

$$
X^{M}(t)=x^{M}(0) \exp \left(\alpha^{M} t+\left(\pi^{T}\right)^{\prime} \sigma W(t)\right)
$$

where $\alpha^{M} \equiv r+\left(\pi^{T}\right)^{\prime}(\mu-r \mathbf{1})-0.5\left\|\left(\pi^{T}\right)^{\prime} \sigma\right\|^{2}$. On the other hand, the dynamics of the GOP are given by

$$
H^{-1}(t)=\exp \left(\alpha^{G} t+\left(\pi^{G}\right)^{\prime} \sigma W(t)\right)
$$

where $\alpha^{G} \equiv r+\left(\pi^{G}\right)^{\prime}(\mu-r \mathbf{1})-0.5\left\|\left(\pi^{G}\right)^{\prime} \sigma\right\|^{2}$ and $\pi^{G}=\left(\sigma \sigma^{\prime}\right)^{-1}(\mu-r \mathbf{1})$. Note that $\pi^{G}=\aleph \pi^{T}$, i.e. the proportions of the growth optimal portfolio and the market portfolio only differ by the scaling factor $\aleph$. Combining (31) and (32) we arrive at

$$
H^{-1}(t)=\exp \left(\left\{\alpha^{G}+\aleph\left(r^{M}(t)-\alpha^{M}\right)\right\} t\right),
$$

where $r^{M}(t)=t^{-1} \ln \left(X^{M}(t) / x^{M}(0)\right)$ is the annualized return of the market portfolio. Hence, the value of the market portfolio at time $T$ is a sufficient statistic for the value of the growth optimal portfolio $1 / H(T)$. Consequently, assuming the market to be in equilibrium, the market portfolio can replace the GOP in the quadratic contract (29). Our results are summarized in the proposition following:

\footnotetext{
${ }^{16}$ We emphasize that using equilibrium arguments is not an unusual approach to derive results about how to make portfolio decisions. See, e.g., Black/Littermann (1991).
} 
Proposition 6.1 (Market Portfolio as Benchmark) Assume the financial market to be in equilibrium and the money market to be in zero net supply. Given a constant investment opportunity set, then due to (33) the value of the market portfolio is a sufficient statistic for the value of the GOP. Consequently, if the investor can observe the value of the market portfolio at time $T$, then the agency conflict between her and the manager is solved, i.e. the solutions of the first-best and second-best problems coincide.

To summarize, imposing an equilibrium assumption allows us to use a passive index, i.e. a portfolio with constant numbers of assets, as benchmark.

\section{Incomplete Markets and More General Cost Structures}

In this section we will discuss two straightforward generalizations of our findings. One will be the treatment of a market where the agent (and the investor) are not allowed to invest in certain stocks. The other remark is connected to the consideration of more general cost structures.

(i) Prohibited stocks. The incompleteness of this example consists of the fact that the investor is only allowed to trade in the first $m$ of $n$ stocks. It is equivalent to the incompleteness example given in Ou-Yang (2003) where the dimension of the driving Brownian motion is greater than the number of risky assets. However, this example can easily be dealt with if we realize that the $m$ linear combinations

$$
\sum_{i=1}^{n} \sigma_{i j} W_{i}(t), j=1, . ., m
$$

of the components of the $n$-dimensional Brownian motion $W(t)$ have exactly the same distribution as the $m$ linear combinations

$$
\sum_{i=1}^{m} \nu_{i j} W_{i}^{*}(t), j=1, . ., m
$$

of the components of an $m$-dimensional Brownian motion $W^{*}(t)$ where $\nu$ can be obtained from a Cholesky decomposition of $\sigma_{1} \sigma_{1}^{\prime}$ (with $\sigma_{1}$ denoting the first $m$ rows of $\sigma$ ). Then, we can solve the delegated portfolio problem in a complete market where $\nu$ takes over the role of $\sigma$. The assertions on the dependence of our optimal final wealth on the growth optimal portfolio remain true if we consider the growth optimal portfolio in the restricted market consisting only of the bond and the first $m$ stocks.

(ii) More general cost structures. As long as the costs that the investor's portfolio managing activities cause can be hedged in our market setting via a suitable replication strategy, we can simply think of paying them as the result of a separate investment problem. However, we cannot deal with transaction costs per trade. In 
this situation, not even Merton's portfolio problem can be solved in a closed form. ${ }^{17}$ Therefore, there is no hope for a solution of the delegated portfolio problem in such a setting.

(iii) More incomplete market situations. If there are constraints on the trading strategy of the investor such as upper bounds on the fraction of total wealth invested into the risky securities then there is no simple carrying over of the explicit results in Cvitanic and Karatzas (1992) or in Chapter 4 of Korn (1997). The main reason for this is the non-constant form of the optimal portfolio in the unconstrained delegated portfolio problem. This may be an interesting aspect for future research.

\section{Conclusion}

In this paper we have analyzed the problem of delegating an investor's portfolio decisions to a manager. We have shown that a specific quadratic compensation scheme solves the agency conflict between investor and manager in the sense that the solution of the first-best and the second-best problem coincide. This contract consists of two parts: The first part corresponds to the manager's first-best optimal share of wealth, whereas the second part consists of a penalty term punishing the manager for deviations from the first-best optimal wealth. The quadratic contract can be implemented given the investor can observe the value of the growth optimal portfolio at the end of the investment horizon. Although ex-ante the quadratic contract differs from the first-best compensation scheme, ex-post they are equal implying Pareto optimal risk-sharing between investor and manager. Furthermore, it has been shown that a contract where the penalty term is zero if the manager outperforms the first-best optimal wealth leads to the same results. As a byproduct, we have seen that the answer to the question "Can an agency conflict be avoided?" is positive. If the economy is in equilibrium and the investment opportunity set is assumed to be constant, it is sufficient that the investor can observe the value of the market portfolio instead of the growth optimal portfolio. Hence, in this case a passive index can serve as a benchmark. Throughout the paper we have assumed that investor and manager agree upon the investment opportunity set, i.e. they both use the same model parameters to make their decisions. This resembles a situation where managers are not able to gather superior information and the investor thus wishes to invest in a passively managed fund. It is beyond the scope of this paper to analyze the situation where the manager is assumed to have superior information about the investment opportunity set such as superior estimates of asset returns. If such an assumption could be justified by empirical evidence, it is in itself the focus of ongoing research.

\footnotetext{
${ }^{17}$ See, e.g., Korn (1997), Chapter 5, for an overview on portfolio problems including fixed and/or proportional transaction costs.
} 


\section{Appendix}

\subsection{Proof of Proposition 4.1.}

Before we prove Proposition 4.1, we prove Lemma 9.1 below under the following assumptions:

(A1) $\chi_{P}(y)<\infty$ for all $y \in(0, \infty)$.

(A2) $\tilde{\chi}_{A}(y):=\mathrm{E}\left|U_{A}\left(V_{A}\left(y \frac{1}{1-\lambda} H(T)\right)\right)\right|<\infty$ for all $y \in(0, \infty)$.

For problems of type II where additionally $U_{A}^{\prime}(0)<\infty$ or $U_{P}^{\prime}(0)<\infty$ we also impose the following condition

(A3) If $U_{A}^{\prime}(0)<\infty$ or $U_{P}^{\prime}(0)<\infty$ then for every fixed $k \in \mathbb{R}$ we assume the relation

$$
P(\ln (H(T))<k)>0
$$

to hold.

The following Lemma shows that the choices are of $y_{1}$ and $y_{2}$ are uniquely determined under these assumptions. We wish to stress that the following results are proved for utility functions in the sense of Definition 3.1 as well as Definition 3.2.

Lemma 9.1 Assume (A1) and (A2) to hold. For problems of type II with $U_{A}^{\prime}(0)<$ $\infty$ or $U_{P}^{\prime}(0)<\infty$ assumption $(A 3)$ is made as well. Then $\chi_{P}$ and $\chi_{A}$ are

(i) continuous on $(0, \infty)$,

(ii) strictly monotonuous decreasing with

(iii) $\chi_{P}(0)=\infty, \chi_{A}(0)=U_{A}(\infty)$.

Furthermore, we obtain

(iv) $\chi_{P}(\infty)=0, \chi_{A}(\infty)=U_{A}(0)$ for utility functions of type $I$ and $\chi_{P}(\infty)=l$, $\chi_{A}(\infty)=\mathcal{U}_{A}(l)$ for utility functions of type II.

Remarks. a) Note that for $U_{A} \geq 0$ or $U_{A} \leq 0$ the condition $\tilde{\chi}_{A}(y)<\infty$ for all $y \in(0, \infty)$ is equivalent to $\left|\chi_{A}(y)\right|<\infty$ for all $y \in(0, \infty)$.

b) Since $V_{P} \geq 0$ we have $\chi_{P} \geq 0$. In contrast to that the function $\chi_{A}$ may posess positve and negative values depending on the range of $U_{A}$. As long as $U_{A}$ is bounded from below or above, without loss of generality we can assume $U_{A} \geq 0$ or $U_{A} \leq 0$, respecively, because utility functions are only uniquely determined up to positive linear transformations. However, the proof of the above properties is a bit more involved for utility functions with a range being equal to the real line such as the logarithmic utility function.

c) Note that (34) is satisfied if $r$ and $\theta$ are uniformly bounded and $\theta$ is deterministic because the support of the then normally distributed Ito-Integral involved in $H$ equals the real line.

Proof of Lemma 9.1. The stated properties of $\chi_{P}$ are proved in Korn (1997) for utility functions of type I. The proofs for utility functions of type II can be carried 
out in a similar way. Hence, we have to verify (i) throught (iv) for $\chi_{A}$. The proofs of (i) and (iii) are the same for utility functions of type I and II. Since we have not restricted the range of $U_{A}$ we need to consider the general case where $U_{A}$ is neither bounded from above nor from below.

(i) Let $y^{\prime} \in(0, \infty)$ and let $\left(y_{n}\right)$ be some sequence with $y_{n} \rightarrow y^{\prime}$. Then for all $\delta$ there exists some $n_{0} \in \mathbb{N}$ such that $\left|y_{n}-y^{\prime}\right| \leq \delta$ for all $n \geq n_{0}$. By the monotony of $U_{A}$ and $V_{A}$, we then get

$\left|U_{A}\left(V_{A}\left(y_{n} \frac{1}{1-\lambda} H(T)\right)\right)\right| \leq\left|U_{A}\left(V_{A}\left(\left(y^{\prime}-\delta\right) \frac{1}{1-\lambda} H(T)\right)\right)\right|+\left|U_{A}\left(V_{A}\left(\left(y^{\prime}+\delta\right) \frac{1}{1-\lambda} H(T)\right)\right)\right|$

for all $n \geq n_{0}$. By assumption, the right hand side is integrable. Hence, by the dominated convergence theorem, we get the claimed continuity.

(ii) We start with utility functions of type I. The claim is proved if

$$
P\left(y \frac{1}{1-\lambda} H(T)<U_{A}^{\prime}(0)\right)>0
$$

for every fixed $y \in(0, \infty)$. Then, since $U_{A}\left(V_{A}\left(y \frac{1}{1-\lambda} H(T)\right)\right)$ is strictly decreasing in $y$ on the set $\left\{y \frac{1}{1-\lambda} H(T)<U_{A}^{\prime}(0)\right\}$ and $U_{A}\left(V_{A}\left(y \frac{1}{1-\lambda} H(T)\right)\right)=U_{A}(0)$ else, the function $\chi_{A}$ is strictly decreasing on $(0, \infty)$. For $U_{A}^{\prime}(0)=\infty$ the relation (35) is obviously satisfied. Otherwise (35) is met due to assumption (34). For utility functions of type II, we always have

$$
P\left(y \frac{1}{1-\lambda} H(T)<U_{A}^{\prime}(l)\right)>0
$$

because $U_{A}^{\prime}(l)=\infty$. Therefore, (ii) also holds for these utility functions.

(iii) Given there exists some $z_{0} \in \mathbb{R}$ such that $U_{A}\left(z_{0}\right)=0$ (if $U_{A} \geq 0$ or $U_{A} \leq 0$ the decomposition via $M_{y}$ is not needed), we define

$$
M_{y}:=\left\{V_{A}\left(y \frac{H(T)}{1-\lambda}\right) \leq z_{0}\right\} .
$$

Then we can decompose $\chi_{A}$ in the following way:

$$
\chi_{A}(y)=\underbrace{\mathrm{E}\left[U_{A}\left(V_{A}\left(y \frac{H(T)}{1-\lambda}\right)\right) 1_{M_{y}}\right]}_{=(*)}+\underbrace{\mathrm{E}\left[U_{A}\left(V_{A}\left(y \frac{H(T)}{1-\lambda}\right)\right) 1_{\bar{M}_{y}}\right]}_{=(* *)},
$$

where $\bar{M}_{y}$ denotes the compelement of $M_{y}$. First recall that for $y \rightarrow 0$ we obtain $V_{A}\left(y \frac{H(T)}{1-\lambda}\right) \rightarrow \infty$ and thus $U_{A}\left(V_{A}\left(y \frac{H(T)}{1-\lambda}\right)\right) \rightarrow U_{A}(\infty)$. Hence, by the monotone convergence theorem, we obtain that $(* *) \rightarrow U_{A}(\infty)$. On the other hand, for $(*)$ we choose some $\bar{y} \in(0, \infty)$. Then for all $y \in(0, \bar{y})$

$$
U_{A}\left(V_{A}\left(\bar{y} \frac{H(T)}{1-\lambda}\right)\right) 1_{M_{y}} \leq U_{A}\left(V_{A}\left(y \frac{H(T)}{1-\lambda}\right)\right) 1_{M_{y}} \leq 0
$$

implying $\left|U_{A}\left(V_{A}\left(\bar{y} \frac{H(T)}{1-\lambda}\right)\right)\right| \geq\left|U_{A}\left(V_{A}\left(y \frac{H(T)}{1-\lambda}\right)\right)\right| 1_{M_{y}}$. Hence, by assumption (A2) and the dominated convergence theorem, we obtain $(*) \rightarrow 0$ and thus $\chi_{A}(y) \rightarrow$ $U_{A}(\infty)$ for $y \rightarrow 0$.

(iv) Consider again decomposition (36). We start with utility functions of type I. Recall that for $y \rightarrow \infty$ we obtain $V_{A}\left(y \frac{H(T)}{1-\lambda}\right) \rightarrow 0$ and thus $U_{A}\left(V_{A}\left(y \frac{H(T)}{1-\lambda}\right)\right) \rightarrow$ 
$U_{A}(0)$. Multiplying $(*)$ by -1 and applying the monotone convergence theorem we obtain $-(*) \rightarrow-U_{A}(0)$ and thus $(*) \rightarrow U_{A}(0)$ for $y \rightarrow \infty$. For utilty functions of type II we get $(*) \rightarrow U_{A}(l)$. It remains to verify that $(* *) \rightarrow 0$ for $y \rightarrow \infty$. Choose $y \in(0, \infty)$. Similar as in the proof of (iii) we obtain

$$
\left|U_{A}\left(V_{A}\left(y \frac{H(T)}{1-\lambda}\right)\right) 1_{\bar{M}_{y}}\right| \leq\left|U_{A}\left(V_{A}\left(\bar{y} \frac{H(T)}{1-\lambda}\right)\right)\right|
$$

for all $y \in(\bar{y}, \infty)$. By assumption (A2), we can thus apply the dominated convergence theorem leading to $(* *) \rightarrow 0$ for $y \rightarrow \infty$.

Proof of Proposition 4.1. By definition of $Z_{A}^{*}$ and $Z_{P}^{*}$ the constraints (8) and (9) are satisfied as equality. Thus the expected utility of the agent is well-defined. Analoguous to Korn (1997) one can show that the expected utility of the prinicipal is well-defined. Further, by Lemma 9.1, we have $y_{A}^{*}>0$. It remains to show that $X^{*}$, $\pi^{*}$, and $I^{*}$ are optimal. Let $\pi$ be some other strategy leading to the terminal wealth $X^{\pi}$ and let $I$ be some other fee. Further, $Z_{A}:=I-C$ and $Z_{P}:=X^{\pi}-I$. Without loss of generality we assume that the participation constraint and constraint (9) are met as equality, i.e.

$$
\mathrm{E}\left[U_{A}\left(Z_{A}^{*}(T)\right)\right]=\mathrm{E}\left[U_{A}\left(Z_{A}(T)\right)\right] \quad \text { and } \quad \mathrm{E}\left[H(T) X^{*}(T)\right]=\mathrm{E}\left[H(T) X^{\pi}(T)\right] .
$$

Due to (2), we have

$$
U_{P}\left(Z_{P}^{*}\right) \geq U_{P}\left(Z_{P}\right)+y_{P}^{*} \frac{1}{1-\lambda} H \cdot\left\{Z_{P}^{*}-Z_{P}\right\} .
$$

By (37), taking expectations leads to

$$
\mathrm{E}\left[U_{P}\left(Z_{P}^{*}(T)\right)\right] \geq \mathrm{E}\left[U_{P}\left(Z_{P}(T)\right)\right]+y_{P}^{*} \frac{1}{1-\lambda}\left\{\mathrm{E}[H(T) I(T)]-\mathrm{E}\left[H(T) I^{*}(T)\right]\right\} .
$$

Hence, it sufficient to show that $\mathrm{E}[H(T) I(T)]-\mathrm{E}\left[H(T) I^{*}(T)\right] \geq 0$. Applying (2) to $U_{A}$ we obtain

$$
\mathrm{E}\left[U_{A}\left(Z_{A}^{*}(T)\right)\right] \geq \mathrm{E}\left[U_{A}\left(Z_{P}(T)\right)\right]+y_{A}^{*} \frac{1}{1-\lambda}\left\{\mathrm{E}[H(T) I(T)]-\mathrm{E}\left[H(T) I^{*}(T)\right]\right\} .
$$

By (37), we conclude

$$
0 \geq y_{A}^{*} \frac{1}{1-\lambda}\left\{\mathrm{E}[H(T) I(T)]-\mathrm{E}\left[H(T) I^{*}(T)\right]\right\} .
$$

Since $y_{A}^{*} \frac{1}{1-\lambda}>0$ the proposition is proved. 


\section{References}

Admati, A. R.; P. Pfleiderer (1997): Does it all add up? Benchmarks and the compensation of active portfolio managers, Journal of Business 70, 323-350.

Amershi, A. H.; J. H. W. Stoeckenius (1983): The theory of syndicates and linear sharing rules, Econometrica 51, 1407-1416.

Black, F.; R. Littermann (1992): Global Portfolio Optimization, Financial Analysts Journal, September-October issue.

Bhattacharya, S.; P. Pfleiderer (1985): Delegated portfolio management, Journal of Economic Theory 36, 1-25.

Borch, K. (1960): The safety loading of reinsurance premiums, Skandinavisk Aktuarietidskrift, 163-184.

Borch, K. (1962): Equilibrium in a reinsurance market, Econometrica 30, 424-444.

Brennan, M. J. (1993): Agency and asset pricing, Working Paper, UCLA.

Cox, J. C.; C. F. Huang (1989): Optimal consumption and portfolio policies when asset prices follow a diffusion process, Journal of Economic Theory 49, 33-83.

Cox, J. C.; C. F. Huang (1991): A variational problem arising in financial economics, Journal of Mathematical Economics 20, 465-487.

Karatzas, I.; J. P. Lehoczky; S. E. Shreve (1987): Optimal portfolio and consumption decisions for a small investor on a finite horizon, SIAM Journal on Control and Optimization 27, 1157-1186.

Cademillas, A., J. Cvitanic, F. Zapatero (2004): Dynamic principal-agent problems with perfect information, Working Paper, USC, Los Angeles.

Cvitanic, J., X. Wan, J. Zhang (2004): Continuous-time principal-agent problems: Necessary and sufficient conditions for optimality, Working Paper, USC, Los Angeles.

Dybvig, P.; H. K. Farnsworth; J. N. Carpenter (2001): Portfolio performance and agency, Working Paper, Washington University in Saint Louis.

Heinkel, R.; N. M. Stoughton (1994): The dynamics of portfolio management contracts, Review of Financial Studies 7, 351-387.

Holmstrom, B. (1979): Moral hazard and observability, Bell Journal of Economics 10, 74-91.

Holmstrom, B; P. Milgrom (1987): Aggregation and linearity in the provision of intertemporal incentives, Econometrica 55, 303-328.

Kihlstrom, R. (1988): Optimal contracts for security analysts and portfolio managers, Studies in Banking and Finance 5, 291-325.

Korn, R. (1997): Optimal portfolios, World Scientific, Singapore.

Merton, R. C. (1969): Lifetime portfolio selection under uncertainty: the continuous case, Reviews of Economical Statistics 51, 247-257.

Merton, R. C. (1971): Optimal consumption and portfolio rules in a continuous-time model, Journal of Economic Theory 3, 373-413. Erratum: ebenda 6 (1973), 213-214.

Merton, R. C. (1973): An intertemporal asset pricing model, Econometrica 41, 867- 
887.

Merton, R. C. (1990): Continuous-time finance, Basil Blackwell, Cambridge MA.

Mirlees, J. (1974): Notes on welfare economics, information and uncertainty, in: M.

Balch; D. McFadden, and S. Wu (eds.): Essays in economic behavior under uncertainty, 243-258, North-Holland, Amsterdam.

Mirlees, J. (1976): The optimal structure of authority and incentives within an organization, Bell Journal of Economics 7, 105-131.

Mossin, J. (1973): Theory of Financial Markets, Prentice-Hall, Englewood Cliffs, New Jersay.

Ou-Yang, H. (2003): Optimal contracts in a continuous-time delegated portfolio management problem, Review of Financial Studies 16, 173-208.

Ross, S. (1973): The economic theory of agency: The principal's problem, American Economic Review 63, 135-139.

Ross, S. (2004): Compensation, incentives, and the duality of risk aversion and riskiness, Journal of Finance 59, 207-225.

Roll, R. (1992): A mean/variance analysis of tracking error, Journal of Portfolio Management 8, Summer, 13-22.

Schättler, H.; J. Sung (1993): The first-order approach to the continuous-time principalagent problem with exponential utility, Journal of Economic Theory 61, 331-371.

Shavell, S. (1979): Risk sharing and incentives in the principal and agent relationship, Bell Journal of Economics 10, 55-73.

Sung, J. (1995): Linearity with project selection and controllable diffusion rate in continuoustime principal-agent problems, RAND Journal of Economics 26, 720-743.

Stoughton, N. M. (1993): Moral hazard and the portfolio management problem, Journal of Finance 48, 2009-2028.

Wilson, R. B. (1968): The theory of syndicates, Econometrica 36, 119-132.

Zender, J. (1987): Portfolio performance measures as managerial compensation, Working Paper, Yale University. 